\title{
Cytosine but not adenine base editor generates mutations in mice
}

\author{
Hye Kyung Lee ${ }^{1, \dagger, *}$, Harold E. Smith², Chengyu Liu ${ }^{3}$, Michaela Willi1, ${ }^{\dagger}$, * \\ and Lothar Hennighausen ${ }^{1, \dagger}$, *
}

${ }^{1}$ Laboratory of Genetics and Physiology, National Institute of Diabetes and Digestive and Kidney Diseases, US National Institutes of Health, Bethesda, Maryland 20892, USA.

${ }^{2}$ Genomics Core, National Institute of Diabetes and Digestive and Kidney Diseases, US National Institutes of Health, Bethesda, Maryland 20892, USA.

${ }^{3}$ Transgenic Core, National Heart, Lung, and Blood Institute, US National Institutes of Health, Bethesda, Maryland 20892, USA.

†These authors contributed equally to this work.

${ }^{*}$ Correspondence to: H.K.L (hyekyung.lee@nih.gov), M.W.

(collaborations@michaelawilli.com) and L.H (lotharh@niddk.nih.gov) 


\section{ABSTRACT}

Deaminase base editing has emerged as a tool to install or correct point mutations in the genomes of living cells in a wide range of organisms and its ultimate success therapeutically depends on its accuracy. Here we have investigated the fidelity of cytosine base editor 4 (BE4) and adenine base editor (ABE) in mouse embryos using unbiased whole genome sequencing of a family-based trio cohort. We demonstrate that BE4-edited mice carry an excess of single-nucleotide variants and deletions compared to ABE-edited mice and controls.

\section{INTRODUCTION}

Deaminase base editing $(1,2)$ directly converts target $C \cdot G$ base pairs to $T \cdot A$ by cytosine base editors $(C B E)$, or target $A \cdot T$ base pairs to $G \cdot C$ by adenine base editors $(A B E)$, without inducing double-stranded DNA breaks (3). Since the majority of known human pathogenic variants are single nucleotide alterations $(2,4)$, base editing has been heralded as a high-fidelity tool to correct single-nucleotide polymorphisms (SNPs) associated with many human disorders. While exceptional precision is paramount in a quest to correct somatic and in particular germline mutations, recent studies have revealed that CBEs can induce bystander mutations, including deletions, in mouse zygotes (5) and plants (6). In contrast, ABE displays a greater fidelity $(5,7)$.

Whole-genome sequencing (WGS) of base-edited rice (8) and mouse embryos (9) revealed that $\mathrm{BE} 3$, a commonly used $\mathrm{CBE}$, induces a large number of inadvertent base changes throughout the genome, while ABE displayed high fidelity. In a separate study, WGS of BE3-edited sheep did not reveal an obvious increase of off-target mutations (10). Since BE3 can introduce unwanted indels $(1,5)$ and other undesirable base substitutions in addition to C-to-T conversions $(5,7,11)$, the fourth-generation BE4, containing a second uracil glycosylase inhibitor (UGI) domain and optimized linker architectures, appears to have an increased fidelity in vitro (12), in mouse zygotes (5) and in rabbit embryos (13). Off-target effect for BE4 might be expected based on WGS studies that examined offtarget mutations introduced by BE3 and ABE $(8,9)$. However, as different sgRNA, editors, and analysis methods might yield different results $(8,9)$, there is a definitive need for investigating in vivo genomic effects of BE4 in comparison with $\mathrm{ABE}$. Along those lines, 
the original study on WGS of CRISPR/Cas9 edited mice suggested the presence of extensive off-target mutations (14), which was, however, likely the result of an imperfect experimental design as pointed out in editorials (15-19). Further WGS investigations by lyer and colleagues as well as our group $(20,21)$ using trio studies demonstrated that CRISPR/Cas9 does not introduce an excess of off-target mutations. Therefore, it is prudent to examine critical issues of base editing, such as the extent of off-target mutations with a larger number of mice and under several conditions. In this study, we have addressed the question of base editing fidelity and conducted unbiased WGS on a total of 44 BE4- and ABE-edited mice, control mice and their wild-type parents.

\section{RESULTS \& DISCUSSION}

To assess on- and off-target fidelities of the advanced BE4 and ABE in mouse embryos, we conducted a family-based trio WGS study (Figure 1A). Fertilized eggs were injected with BE4 or ABE7.10 mRNA together with a sgRNA used by both editors, which permitted a direct comparison. Two-cell stage embryos were implanted into surrogate mothers and 13 ABE-edited and nine CBE-edited founder mice were born, together with 13 noninjected controls (Figure 1B). All founders (Figure 1C and Supplementary Figure 1) were screened by Sanger sequencing to identify mutants and targeted deep-sequencing was performed to determine haplotypes. ABE introduced A-to-G transitions in the target window and, except one, no bystander or proximal off-target mutations were detected in the 33 edited alleles. In contrast, BE4 induced not only the expected C-to-T transitions but also C-to-G and C-to-A conversions in the target site, frequent proximal off-target mutations, and deletions in four of the nine founders (12 out of the 17 edited alleles) (Figure 1C and Supplementary Figure 1).

Unbiased WGS was performed on the 22 edited mice, 13 controls and nine parents at an average depth of 60x (Figure 1B and Supplementary Table 1). The WGS data were analyzed using GATK with Joint Genotyping and subsequent filtering to identify single nucleotide variants (SNVs) and indels for each individual mouse (Figure 2A). Lumpy with SvTyper was used to identify indels. To explicitly identify de novo mutations, the SNVs and indels present in the parents were subtracted from those identified in the progeny (Supplementary Tables 2 and 3). Non-edited control mice had accumulated an average 
of 132 de novo SNVs (Figure 2B and Supplementary Table 2). On average 119 SNVs were detected in ABE-edited mice, comparable to that in controls. In contrast, BE4-edited mice carried on average $221 \mathrm{SNVs}$, a significant increase (Kruskal-Wallis chi-square: $\mathrm{p}=0.002$ ), especially $\mathrm{C}$-to- $\mathrm{T}$ variants (Figure $2 \mathrm{~B}$, Supplementary Figure 2 and Supplementary Table 2).

About $2 \%$ of off-target SNVs coincided with the predicted off-target site, CRISPR, suggesting that the majority of mutations were not dependent of the sgRNA and predictable by predicted off-target sites (Supplementary Table 4). The increased off-target editing observed in BE4 but not $A B E$ implies that these mutations were the result of cytosine deaminase AID/APOBEC1 which can induce SNVs in the absence of sgRNAs (1). C-to-T conversions (plus some C-to-A and C-to-G) are overrepresented in de novo SNVs observed in BE4-edited mice, consistent with enzymatic activity of BE4. Since four out of the nine BE4-edited mice carried additional deletions proximal to the target region, we analyzed globally their indel frequencies compared with controls (Figure $2 \mathrm{C}$ and Supplementary Table 3). The numbers of indels in the BE4- and ABE-edited group showed no differences from the control group (Figure 2C), also not regarding their characteristics (Supplementary Table 5).

Our results confirm and extend previous work that BE3 but not $A B E$ increases offtarget SNVs in mouse embryos (9) and rice (8) by analyzing a large data set of nine BE4edited and 13 ABE-edited mice (a total of 50 mutant alleles). We observed a significant mutation rate with the improved BE4 in mouse embryos. While base-edited mouse embryos (9) acquire off-targets independent on sgRNAs, in base-edited rice off-target mutations can coincide with predicted off target sites (8) suggesting sgRNA dependence. Thus, using the same sgRNA for both BE4 and ABE eliminates latent promiscuous guide targeting as the explanation for off-target mutations in BE4, a unique aspect of our study. We demonstrated an approximately two-fold increase of de novo offtarget mutations in BE4-edited embryos, which favorably compares to the more than 20fold increase observed in BE3-edited mouse embryos (9). However, given the variation between individual mice, our study and that in rice (8) show a statistically significant difference in off-target SNVs. BE4, an advanced version of BE3, contains an additional UGI (uracil DNA glycosylase inhibitor) and a shortened linker, as a means to enhance its 
specificity (12). Further studies will be needed to validate the higher genome-wide fidelity of BE4. However, BE4 caused inadvertent proximal off-target mutations and deletions in four of the nine founders, which has implications in its use as therapeutic agent. These adverse mutations are likely independent of the sgRNA used as none of the 13 embryos edited with $A B E$ and the same sgRNA displayed any proximal off-target mutations and deletions. It is not clear whether BE3-induced proximal off-target mutations were detected in the two WGS studies $(8,9)$.

In summary, our study emphasizes the high fidelity of $A B E$ as compared to BE4, which also induces increased unwanted base substitutions and deletions in close proximity to the designed target site. Such unwanted mutations are of particular concern when correcting disease-associated SNPs in proteins, as they could adversely alter nontargeted amino acids. Our study also emphasizes the need to monitor off-target mutations in clonal populations, as the analysis of large pools of cells with variable editing, as commonly conducted in in vitro experiments (22-24), results in population averaging. Based on our study and previous experiments $(5,8,9)$, ABE appears to be the current choice for base editing because of its fidelity at target sites and throughout the genome. However, caution about the fidelity of deaminase base editors comes from recent studies that demonstrated extensive off-target RNA editing $(25,26)$.

\section{MATERIALS AND METHODS}

\section{Mice}

All animals were housed and handled according to the guidelines of the Animal Care and Use Committee (ACUC) of the NIH (https://oacu.oir.nih.gov) and all animal experiments were approved by the ACUC of National Institute of Diabetes and Digestive and Kidney Diseases (NIDDK, MD) and performed under the NIDDK animal protocol K089-LGP-17. Base edited founder mice were generated using C57BL/6N mice (Charles River Laboratories, MD) by the Transgenic Core of the National Heart, Lung, and Blood Institute (NHLBI, MD).

\section{CRISPR reagents and microinjection of mouse zygotes}


The Wap-E1 sgRNA (GGCACAGTATGGGCCCTTCT) (27), which contains two cytidines and two adenines near the editing window, was designed and synthesized using ThermoFiser's sgRNA in vitro transcription service. The pCMV-BE4 plasmid (gift from David Liu's laboratory) and pCMV-ABE7.10 plasmid (Addgene plasmid \#102919) were linearized and then their mRNAs were synthesized in vitro using the mMESSAGE mMACHINE T7 kit (ThermoFisher Scientific). Mouse zygotes were produced by in vitro fertilization (IVF) using eggs collected from eight superovulated C57BL/6N female mice and sperm collected from one C57BL/6N male (Charles River Laboratories). The ABE and BE4 mRNAs (50ng/ul) were separately microinjected with the sgRNA (20ng/ul) into the cytoplasm of the IVF zygotes. After culturing overnight in M16 medium, those embryos reached 2-cell stage of development were implanted into oviducts of pseudopregnant foster mothers (Swiss Webster, NY). Mice born to the foster mothers were genotyped and subsequently analyzed by whole genome sequencing.

\section{Genotyping}

Genomic DNA was isolated from the tip of tails of 3-week-old mice using Wizard Genomic DNA purification Kit (Promega), amplified by PCR, and followed by Sanger sequencing (Quintarabio, CA). Mutations were identified by PCR amplifying a 599 bp fragment encompassing the target sequence, followed by Sanger sequencing. Library preparation and whole genome sequencing was conducted by the Broad Institute (Cambridge, MA) using Illumina HiSeq $X$, at a coverage of $60 x$ using 150bp paired-end reads (Supplementary Table 1).

PCR Primers

Wap-S1_F1: GTTGGAACCCATCACAGACAAAGG

Wap-S1_R1: TGTAGAAACAGAGCAGAGAGGTGG

\section{GATK analysis}

WGS (60x) was performed on 44 mice, nine parents (one male and eight females), and their progeny, including 22 founder mice carrying base substitutions at target sites induced by $A B E$ or BE4 using one guide RNA and 13 non-injected control ones. The 
analysis was performed accordingly to the GATK best practices guidelines (28-30) for germline mutations (version 3.8-0). Quality control and alignment was done by BBmap (31) (version 37.36) and BWA MEM (32) (version 0.7.15), respectively, using the reference genome $\mathrm{mm} 10$.

For runtime opitmazation, the aligned BAM files were split up to a chromosome level (for runtime optimization) and reads aligned to different chromosomes were filtered using SAMtools (33) (version 1.5), followed by Picard tools (34) (version 2.9.2) to mark duplicates. The GATK analysis workflow was applied as follows: (i) base recalibration GATK BaseRecalibrator, AnalyzeCovariates, and PrintReads - using the databases of known polymorphic sites, dbSNP142 and MGPv5 (provided by the high-performance computing team of the NIH (Biowulf)); (ii) variant calling - GATK HaplotypeCaller - with the genotyping mode "discovery", the "ERC" parameter for creating gvcf and a minimum phred-scaled confidence threshold of 30 . The final step included merging the VCF files of each chromosome (GenomeAnalysisTK, GATK).

\section{GATK SNV analysis}

Joint genotyping was applied on on all 44 samples together and hard filters were applied: "QD < 2.0 || FS > 60.0 || MQ < 40.0 || MQRankSum < -12.5 || ReadPosRankSum < -8.0 || SOR > 3 ". The resulting SNVs were additionally filtered by removing those overlapping with repetitive elements (35) (UCSC's masked repeats plus simple repeats; http://hgdownload.soe.ucsc.edu/goldenPath/mm10/database) and black regions (ENCODE (36); http://mitra.stanford.edu/kundaje/akundaje/release/blacklists/mm10mouse/). On an individual level, only SNVs with a genotype of $0 / 1$ or $1 / 1$ were kept. Further filtering steps comprised the removal of SNVs with a read depth smaller than 10, an excessive read depth (37) $(d+3 \sqrt{ } d, d=$ average read depth), an allele frequency less than $10 \%$ using a variety of tools (38-40). All SNVs within +/- 5bp of an indel border were removed as likely false-positives.

\section{Simple GATK indel analysis}

Indels identified by GATK where extracted after joint genotyping and subsequently hard filters were applied according to the GATK recommendations: "QD < 2.0 || FS > 200.0 || 
ReadPosRankSum <-20.0 || SOR > 10.0 ". Indels overlapping with repetitive elements

(UCSC's masked

repeats

plus

simple

repeats;

http://hgdownload.soe.ucsc.edu/goldenPath/mm10/database) or black regions (ENCODE(36); http://mitra.stanford.edu/kundaje/akundaje/release/blacklists/mm10mouse/) were removed. The individual samples were filtered keeping only indels with the genotypes of $0 / 1$ and $1 / 1$, removing those with a read depth smaller than 10 as well as sites with an excessive number of reads $(37)(d+3 \sqrt{ } d, d=$ average read depth). Last, all simple indels that overlap with complex indels identified using LUMPY were excluded. For all those steps a variety of tools (38-40) was applied.

\section{Complex indel analysis using Lumpy}

The analysis of complex indels was done on the same samples using Lumpy (41) according to the guidelines. Mapping was done using BWA MEM (32), with the parameters "--excludeDups --addMateTags --maxSplitCount 2 --minNonOverlap 20" (reference genome mm10), followed by Lumpy (41) using the discordant and split reads as input and genotypes were identified using SVTyper (42). The subsequent filtering steps comprised the selection of indels with a genotype of $0 / 1$ and $1 / 1$ and the removal of indels with a quality smaller than 100 and an excessive read coverage ( $d+3 \sqrt{ } d(37)$, where $d$ is the average read depth) or a SU value (Number of pieces of evidence supporting the variant across all samples) smaller than 5 . Indels overlapping with repetitive elements (35) or black regions (36) were excluded.

\section{Statistical analysis}

All statistical analyses were performed with R package 3.3.3 (http://www.R-project.org/). Kruskal-Wallis test was applied using kruskal.test and pairwise comparison was done with a Wilcoxon Rank Sum wilcox.test in R. All values represent means \pm S.D.

\section{Targeted deep-seq}

Target sites were amplified from mouse genomic DNA using Phusion polymerase (Thermo Fisher Scientific) and PCR products were prepared as libraries for next- 
generation sequencing. Pooled PCR amplicons were conducted paired-end sequencing using an Illumina MiSeq (Illumina).

PCR Primers

Wap-S1_F2: AAGACAGGAGGTTTTGAGCAAGGC

Wap-S1_R2: CACCAGTGAAGACAAAGGAGTATGG

\section{Off-target analysis}

Off-target sites were predicted using http://crispor.tefor.net/(43). The resulting off-target sites were filtered using the same criteria as for SNVs and indels, to consider only those areas of the genome which do not coincide with black regions (36) (ENCODE (36); http://mitra.stanford.edu/kundaje/akundaje/release/blacklists/mm10-mouse) or repetitive elements (35) (UCSC's masked repeats plus simple repeats; http://hgdownload.soe.ucsc.edu/goldenPath/mm10/database). Mutations, which were present in the population and not only in base-edited mice, but identified at predicted offtarget sites, were not considered as a consequence of base editing.

\section{Data availability}

The data are available at SRA under project number PRJNA555149.

\section{ACKNOWLEDGMENTS}

This work utilized the computational resources of the NIH HPC Biowulf cluster (http://hpc.nih.gov). This work was supported by the Intramural Research Programs (IRPs) of National Institute of Diabetes and Digestive and Kidney Diseases (NIDDK) and National Heart, Lung, and Blood Institute (NHLBI).

\section{FUNDING}

This study was supported by the IRPs of NHLBI and NIDDK.

\section{AUTHOR CONTRIBUTIONS}


All authors designed the study. C.L. generated mutant mice. H.K.L. identified and characterized mutant mice. H.K.L. performed experiments and data analysis. M.W. performed computational analysis. H.K.L., M.W. and L.H. supervised the study. H.K.L., M.W. and L.H. wrote the manuscript and all authors approved the final version.

\section{Competing interests}

The authors have not competing interests.

\section{REFERENCES}

1. Komor, A.C., Kim, Y.B., Packer, M.S., Zuris, J.A. and Liu, D.R. (2016) Programmable editing of a target base in genomic DNA without double-stranded DNA cleavage. Nature, 533, 420-424.

2. Gaudelli, N.M., Komor, A.C., Rees, H.A., Packer, M.S., Badran, A.H., Bryson, D.I. and Liu, D.R. (2017) Programmable base editing of $A^{*} T$ to $G^{*} C$ in genomic DNA without DNA cleavage. Nature, 551, 464-471.

3. Komor, A.C., Badran, A.H. and Liu, D.R. (2018) Editing the Genome Without Double-Stranded DNA Breaks. ACS Chem Biol, 13, 383-388.

4. Landrum, M.J., Lee, J.M., Benson, M., Brown, G., Chao, C., Chitipiralla, S., Gu, B., Hart, J., Hoffman, D., Hoover, J. et al. (2016) ClinVar: public archive of interpretations of clinically relevant variants. Nucleic Acids Res, 44, D862-868.

5. Lee, H.K., Willi, M., Miller, S.M., Kim, S., Liu, C., Liu, D.R. and Hennighausen, L. (2018) Targeting fidelity of adenine and cytosine base editors in mouse embryos. Nat Commun, 9, 4804.

6. Shimatani, Z., Kashojiya, S., Takayama, M., Terada, R., Arazoe, T., Ishii, H., Teramura, H., Yamamoto, T., Komatsu, H., Miura, K. et al. (2017) Targeted base editing in rice and tomato using a CRISPR-Cas9 cytidine deaminase fusion. Nat Biotechnol, 35, 441-443.

7. Liu, Z., Lu, Z., Yang, G., Huang, S., Li, G., Feng, S., Liu, Y., Li, J., Yu, W., Zhang, Y. et al. (2018) Efficient generation of mouse models of human diseases via ABEand BE-mediated base editing. Nat Commun, 9, 2338. 
8. Jin, S., Zong, Y., Gao, Q., Zhu, Z., Wang, Y., Qin, P., Liang, C., Wang, D., Qiu, J.L., Zhang, F. et al. (2019) Cytosine, but not adenine, base editors induce genome-wide off-target mutations in rice. Science, 364, 292-295.

9. Zuo, E., Sun, Y., Wei, W., Yuan, T., Ying, W., Sun, H., Yuan, L., Steinmetz, L.M., Li, Y. and Yang, H. (2019) Cytosine base editor generates substantial off-target single-nucleotide variants in mouse embryos. Science, 364, 289-292.

10. Zhou, S., Cai, B., He, C., Wang, Y., Ding, Q., Liu, J., Liu, Y., Ding, Y., Zhao, X., Li, G. et al. (2019) Programmable Base Editing of the Sheep Genome Revealed No Genome-Wide Off-Target Mutations. Front Genet, 10, 215.

11. Kim, K., Ryu, S.M., Kim, S.T., Baek, G., Kim, D., Lim, K., Chung, E., Kim, S. and Kim, J.S. (2017) Highly efficient RNA-guided base editing in mouse embryos. Nat Biotechnol, 35, 435-437.

12. Komor, A.C., Zhao, K.T., Packer, M.S., Gaudelli, N.M., Waterbury, A.L., Koblan, L.W., Kim, Y.B., Badran, A.H. and Liu, D.R. (2017) Improved base excision repair inhibition and bacteriophage Mu Gam protein yields C:G-to-T:A base editors with higher efficiency and product purity. Sci Adv, 3, eaao4774.

13. Liu, Z., Chen, M., Chen, S., Deng, J., Song, Y., Lai, L. and Li, Z. (2018) Highly efficient RNA-guided base editing in rabbit. Nat Commun, 9, 2717.

14. Schaefer, K.A., Wu, W.H., Colgan, D.F., Tsang, S.H., Bassuk, A.G. and Mahajan, V.B. (2017) Unexpected mutations after CRISPR-Cas9 editing in vivo. Nat Methods, 14, 547-548.

15. Nutter, L.M.J., Heaney, J.D., Lloyd, K.C.K., Murray, S.A., Seavitt, J.R., Skarnes, W.C., Teboul, L., Brown, S.D.M. and Moore, M. (2018) Response to "Unexpected mutations after CRISPR-Cas9 editing in vivo". Nat Methods, 15, 235-236.

16. Wilson, C.J., Fennell, T., Bothmer, A., Maeder, M.L., Reyon, D., Cotta-Ramusino, C., Fernandez, C.A., Marco, E., Barrera, L.A., Jayaram, H. et al. (2018) Response to "Unexpected mutations after CRISPR-Cas9 editing in vivo". Nat Methods, 15, 236-237.

17. Lescarbeau, R.M., Murray, B., Barnes, T.M. and Bermingham, N. (2018) Response to "Unexpected mutations after CRISPR-Cas9 editing in vivo". Nat Methods, 15, 237. 
18. Lareau, C.A., Clement, K., Hsu, J.Y., Pattanayak, V., Joung, J.K., Aryee, M.J. and Pinello, L. (2018) Response to "Unexpected mutations after CRISPR-Cas9 editing in vivo". Nat Methods, 15, 238-239.

19. Kim, S.T., Park, J., Kim, D., Kim, K., Bae, S., Schlesner, M. and Kim, J.S. (2018) Response to "Unexpected mutations after CRISPR-Cas9 editing in vivo". Nat Methods, 15, 239-240.

20. Willi, M., Smith, H.E., Wang, C., Liu, C. and Hennighausen, L. (2018) Mutation frequency is not increased in CRISPR-Cas9-edited mice. Nat Methods, 15, 756758.

21. lyer, V., Boroviak, K., Thomas, M., Doe, B., Riva, L., Ryder, E. and Adams, D.J. (2018) No unexpected CRISPR-Cas9 off-target activity revealed by trio sequencing of gene-edited mice. PLoS Genet, 14, e1007503.

22. Kim, D., Lim, K., Kim, S.T., Yoon, S.H., Kim, K., Ryu, S.M. and Kim, J.S. (2017) Genome-wide target specificities of CRISPR RNA-guided programmable deaminases. Nat Biotechnol, 35, 475-480.

23. Kim, D., Kim, D.E., Lee, G., Cho, S.I. and Kim, J.S. (2019) Genome-wide target specificity of CRISPR RNA-guided adenine base editors. Nat Biotechnol, 37, 430435.

24. Yang, L., Briggs, A.W., Chew, W.L., Mali, P., Guell, M., Aach, J., Goodman, D.B., Cox, D., Kan, Y., Lesha, E. et al. (2016) Engineering and optimising deaminase fusions for genome editing. Nat Commun, 7, 13330.

25. Grunewald, J., Zhou, R., Garcia, S.P., Iyer, S., Lareau, C.A., Aryee, M.J. and Joung, J.K. (2019) Transcriptome-wide off-target RNA editing induced by CRISPR-guided DNA base editors. Nature, 569, 433-437.

26. Zhou, C., Sun, Y., Yan, R., Liu, Y., Zuo, E., Gu, C., Han, L., Wei, Y., Hu, X., Zeng, R. et al. (2019) Off-target RNA mutation induced by DNA base editing and its elimination by mutagenesis. Nature.

27. Shin, H.Y., Willi, M., HyunYoo, K., Zeng, X., Wang, C., Metser, G. and Hennighausen, L. (2016) Hierarchy within the mammary STAT5-driven Wap super-enhancer. Nat Genet, 48, 904-911. 
28. McKenna, A., Hanna, M., Banks, E., Sivachenko, A., Cibulskis, K., Kernytsky, A., Garimella, K., Altshuler, D., Gabriel, S., Daly, M. et al. (2010) The Genome Analysis Toolkit: a MapReduce framework for analyzing next-generation DNA sequencing data. Genome Res, 20, 1297-1303.

29. DePristo, M.A., Banks, E., Poplin, R., Garimella, K.V., Maguire, J.R., Hartl, C., Philippakis, A.A., del Angel, G., Rivas, M.A., Hanna, M. et al. (2011) A framework for variation discovery and genotyping using next-generation DNA sequencing data. Nat Genet, 43, 491-498.

30. Van der Auwera, G.A., Carneiro, M.O., Hartl, C., Poplin, R., Del Angel, G., LevyMoonshine, A., Jordan, T., Shakir, K., Roazen, D., Thibault, J. et al. (2013) From FastQ data to high confidence variant calls: the Genome Analysis Toolkit best practices pipeline. Curr Protoc Bioinformatics, 43, 11 10 11-33.

31. Bushnell, B. (2016).

32. Li, H. and Durbin, R. (2009) Fast and accurate short read alignment with BurrowsWheeler transform. Bioinformatics, 25, 1754-1760.

33. Li, H., Handsaker, B., Wysoker, A., Fennell, T., Ruan, J., Homer, N., Marth, G., Abecasis, G., Durbin, R. and Genome Project Data Processing, S. (2009) The Sequence Alignment/Map format and SAMtools. Bioinformatics, 25, 2078-2079.

34. Broad Institute. (2016).

35. Casper, J., Zweig, A.S., Villarreal, C., Tyner, C., Speir, M.L., Rosenbloom, K.R., Raney, B.J., Lee, C.M., Lee, B.T., Karolchik, D. et al. (2018) The UCSC Genome Browser database: 2018 update. Nucleic Acids Res, 46, D762-D769.

36. Consortium, E.P. (2012) An integrated encyclopedia of DNA elements in the human genome. Nature, 489, 57-74.

37. $\mathrm{Li}, \mathrm{H}$. (2014) Toward better understanding of artifacts in variant calling from highcoverage samples. Bioinformatics, 30, 2843-2851.

38. Quinlan, A.R. and Hall, I.M. (2010) BEDTools: a flexible suite of utilities for comparing genomic features. Bioinformatics, 26, 841-842.

39. Neph, S., Kuehn, M.S., Reynolds, A.P., Haugen, E., Thurman, R.E., Johnson, A.K., Rynes, E., Maurano, M.T., Vierstra, J., Thomas, S. et al. (2012) BEDOPS: highperformance genomic feature operations. Bioinformatics, 28, 1919-1920. 
40. Danecek, P., Auton, A., Abecasis, G., Albers, C.A., Banks, E., DePristo, M.A., Handsaker, R.E., Lunter, G., Marth, G.T., Sherry, S.T. et al. (2011) The variant call format and VCFtools. Bioinformatics, 27, 2156-2158.

41. Layer, R.M., Chiang, C., Quinlan, A.R. and Hall, I.M. (2014) LUMPY: a probabilistic framework for structural variant discovery. Genome Biol, 15, R84.

42. Chiang, C., Layer, R.M., Faust, G.G., Lindberg, M.R., Rose, D.B., Garrison, E.P., Marth, G.T., Quinlan, A.R. and Hall, I.M. (2015) SpeedSeq: ultra-fast personal genome analysis and interpretation. Nat Methods, 12, 966-968.

43. Haeussler, M., Schonig, K., Eckert, H., Eschstruth, A., Mianne, J., Renaud, J.B., Schneider-Maunoury, S., Shkumatava, A., Teboul, L., Kent, J. et al. (2016) Evaluation of off-target and on-target scoring algorithms and integration into the guide RNA selection tool CRISPOR. Genome Biol, 17, 148.

Figure legends

Figure 1. Base-editing by BE4 and ABE in mouse embryos. (A) Experimental design of the family-based trio study. (B) Summary of data obtained from mouse zygotes coinjected with $A B E$ or BE4 mRNA and a sgRNA. (C) Alignment of sequences from founder mice. 33 mutant alleles edited by $A B E$, and 17 alleles edited by BE4 were analyzed. The sgRNA sequence is underlined and the PAM sites shown in green. The target nucleotides and edited nucleotide are shown in bold black and blue, respectively. Unintended nucleotide substitutions are shown in red. Deletions are marked with a deletion symbol.

\section{Figure 2. De novo mutation frequencies in base-edited and control mice compared} to their parents. (A) Workflow of whole genome sequencing analysis for SNVs and indels. (b and c) Numbers of de novo SNVs (B) and indels (C) identified in mutants and controls. Boxplot: center line, mean; box limits, plus and minus standard deviation; whiskers, minimum and maximum. Wilcoxon rank-sum test and Mann-Whitney-U-Test for the pairwise comparison were used to evaluate the statistical significance of differences between groups. 


\section{Supplementary Figures}

Supplementary Figure 1. Alignments of mutant sequences from founder mice carrying base-edited mutations. The sgRNA is underlined and the corresponding PAM sequence is shown in green. Within the editing window, the target nucleotides for base editing and nucleotides substituted by base editing are shown in bold black and blue, respectively. Bystanders are highlighted in red and deletions are indicated using a deletion symbol. WT, wild-type.

Supplementary figure 2. The frequencies of different types of total $(A)$ and de novo SNVs (B) in individuals edited by the base editors and in the control group. 
Figure 1.

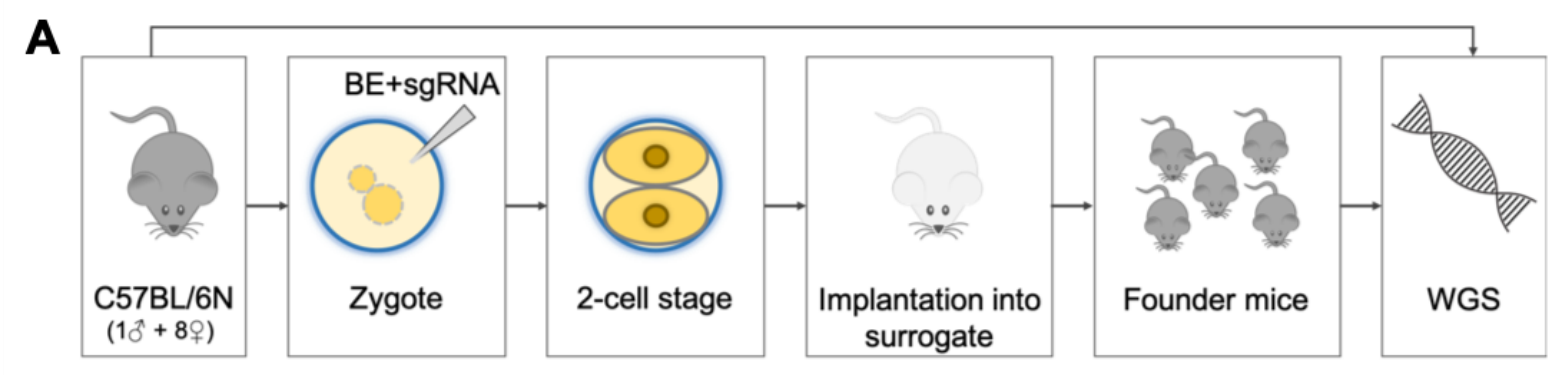

B

\begin{tabular}{|c|c|c|c|c|c|}
\hline $\begin{array}{c}\text { No. of } \\
\text { Parents }\end{array}$ & Base editor & $\begin{array}{c}\text { No. of } \\
\text { injected } \\
\text { embryos }\end{array}$ & $\begin{array}{c}\text { No. of } \\
\text { embryos } \\
\text { implanted }\end{array}$ & $\begin{array}{c}\text { No. of pups } \\
\text { born }\end{array}$ & $\begin{array}{c}\text { Mutation } \\
\text { frequency }\end{array}$ \\
\hline \multirow{2}{*}{$\begin{array}{c}10 \\
8\end{array}$} & Control & - & 68 & 13 & - \\
\cline { 2 - 6 } & ABE & 196 & 124 & 13 & 13 \\
\cline { 2 - 6 }$\left(\begin{array}{c}200 \text { pooled } \\
\text { zygotes })\end{array}\right.$ & BE4 & 170 & 120 & 9 & 9 \\
\hline
\end{tabular}

C

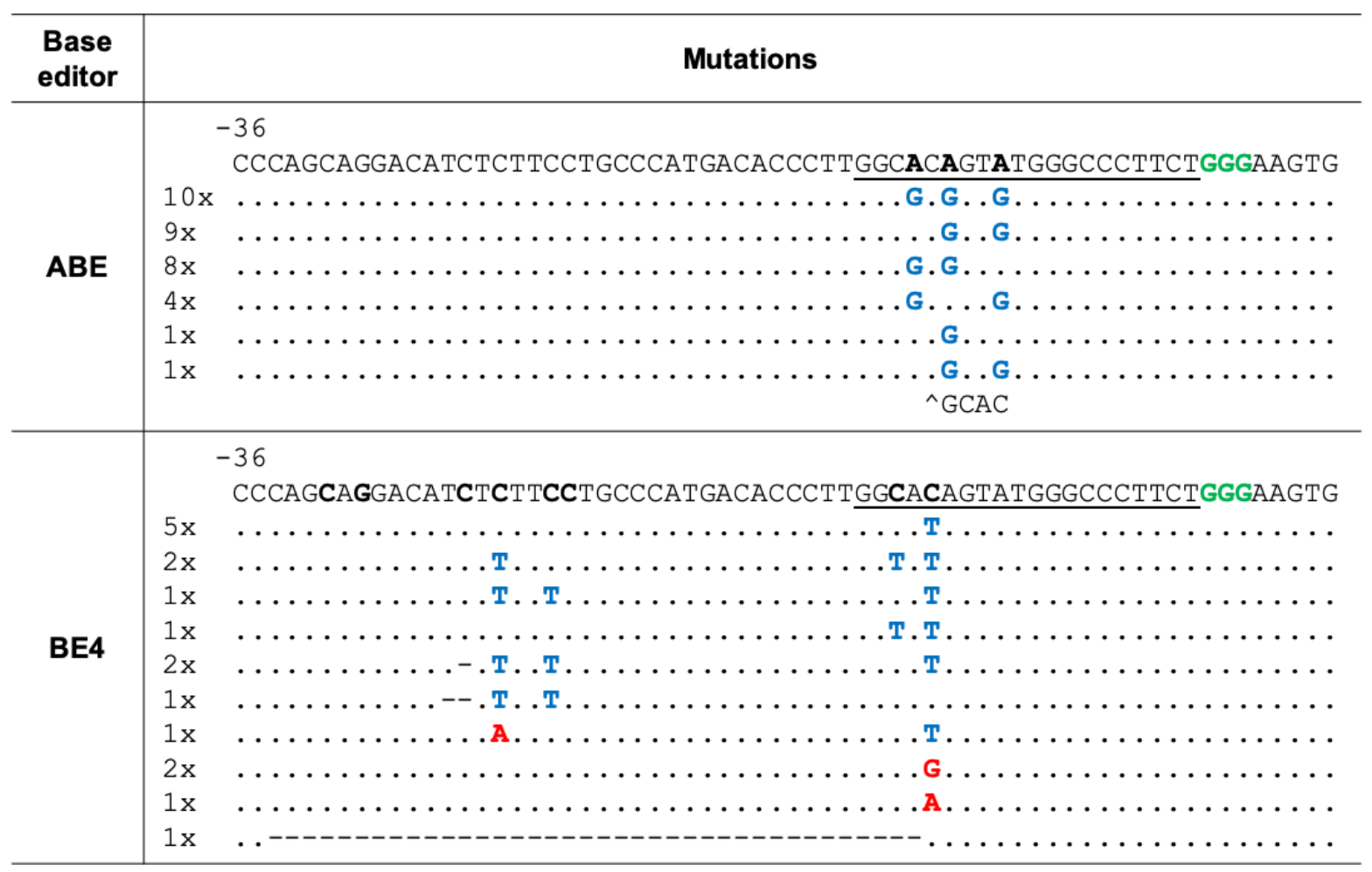


Figure 2.

A

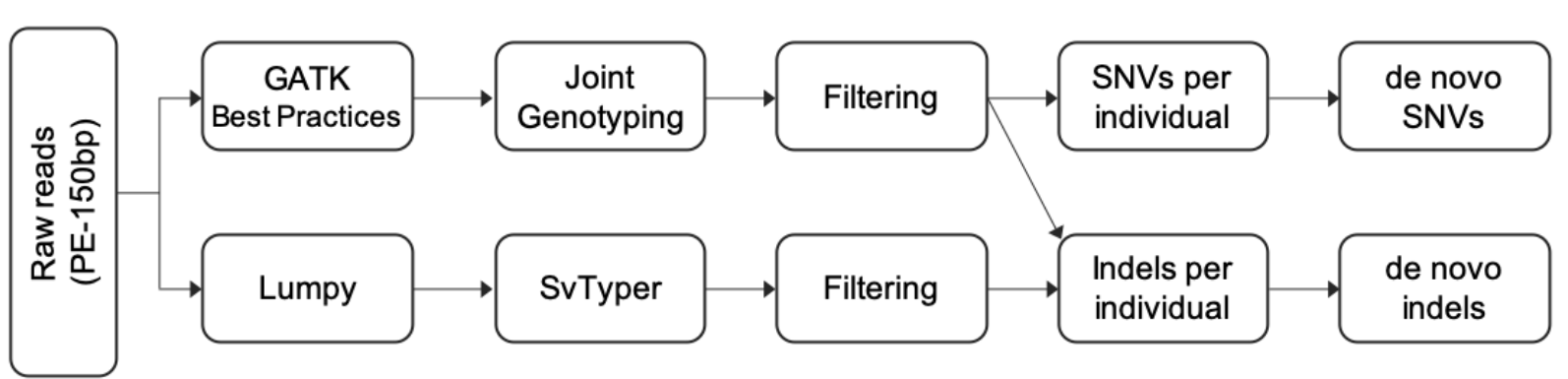

B

de novo SNVs

Kruskal-Wallis chi-squared:

$p$-value $=0.002002$

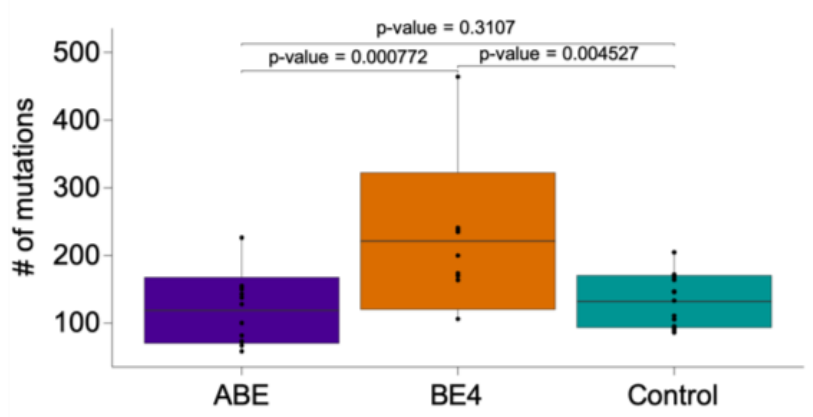

C de novo Indels

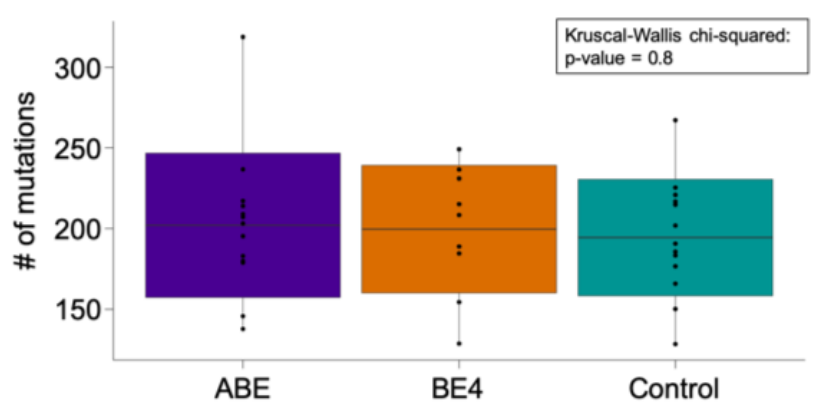


bioRxiv preprint doi: https://doi.org/10.1101/731927; this version posted August 12,2019 . The copyright holder for this preprint (which was not certified by peer review) is the author/funder. All rights reserved. No reuse allowed without permission.

\section{Supplementary Figure 1.}

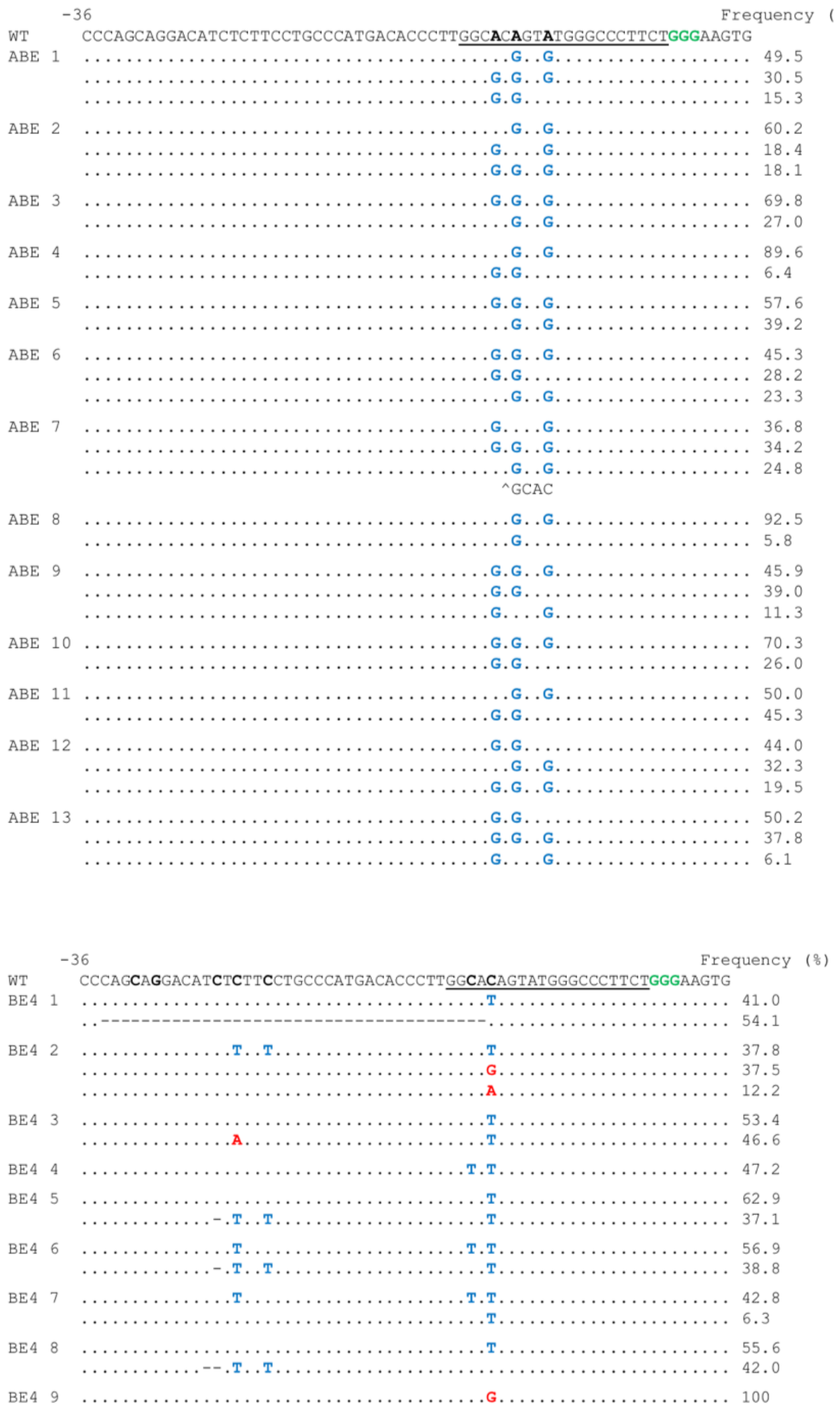




\section{Supplementary Figure 2.}

A

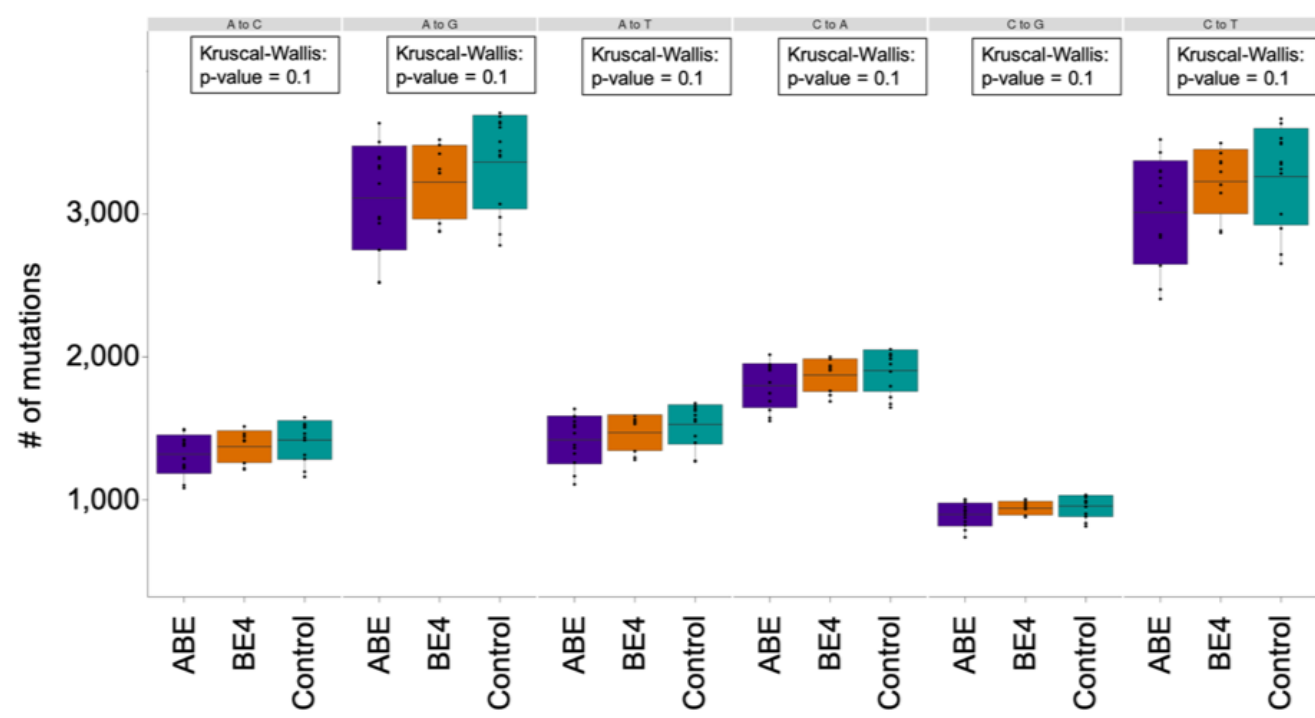

B

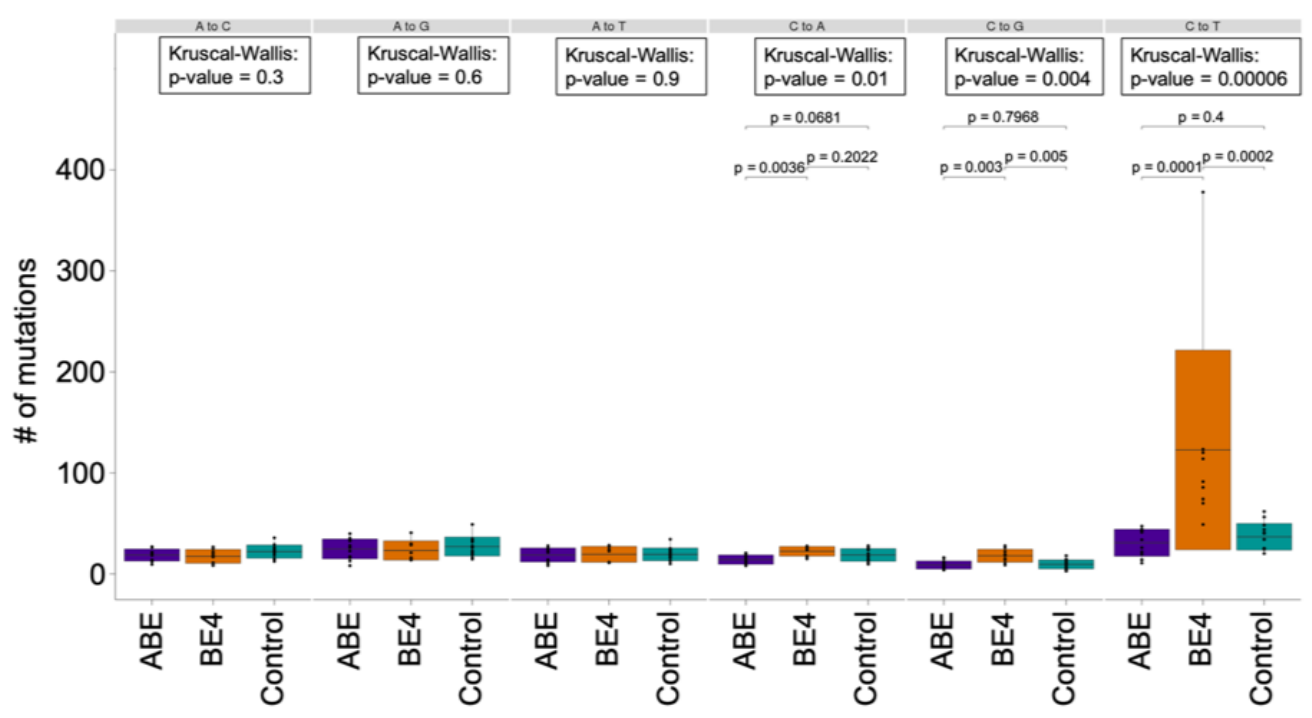




\section{Supplementary Table 1 Raw read counts of the WGS samples}

\begin{tabular}{|l|c|r|}
\hline \multicolumn{3}{|c|}{ Parents } \\
\hline Sample & Gender & Raw Read Counts \\
\hline Parent 1 & & $707,581,699$ \\
Parent 2 & & $868,778,178$ \\
Parent 3 & & $1,072,332,421$ \\
Parent 4 & & $1,162,951,335$ \\
Parent 5 & $\uparrow$ & $767,582,455$ \\
Parent 6 & & $772,091,887$ \\
Parent 7 & & $823,878,962$ \\
Parent 8 & & $783,498,969$ \\
\hline Parent 9 & o & $869,713,827$ \\
\hline
\end{tabular}

\begin{tabular}{|c|c|c|}
\hline \multicolumn{3}{|c|}{ ABE edited Progeny } \\
\hline Sample & Gender & Raw Read Counts \\
\hline ABE 1 & & $769,235,486$ \\
\hline ABE 2 & & $998,329,339$ \\
\hline ABE 3 & 0 & $1,033,798,370$ \\
\hline ABE 4 & & $808,263,614$ \\
\hline ABE 5 & & $651,033,776$ \\
\hline ABE 6 & & $839,652,961$ \\
\hline ABE 7 & & $1,137,405,701$ \\
\hline ABE 8 & A & $735,637,641$ \\
\hline ABE 9 & $\delta$ & $919,901,911$ \\
\hline ABE 10 & & $881,365,098$ \\
\hline ABE 11 & & $686,200,552$ \\
\hline ABE 12 & $N / A$ & $1,141,706,029$ \\
\hline ABE 13 & $\mathrm{~N} / \mathrm{A}$ & 874.997 .141 \\
\hline
\end{tabular}

\begin{tabular}{|c|c|c|}
\hline \multicolumn{3}{|c|}{ Control Progeny } \\
\hline Sample & Gender & Raw Read Counts \\
\hline Control 1 & & $945,102,788$ \\
\hline Control 2 & & $945,102,788$ \\
\hline Control 3 & + & $945,102,788$ \\
\hline Control 4 & & $914,556,327$ \\
\hline Control 5 & & $767,715,791$ \\
\hline Control 6 & & $935,639,522$ \\
\hline Control 7 & & $651,111,242$ \\
\hline Control 8 & & $669,733,921$ \\
\hline Control 9 & & $671,835,774$ \\
\hline Control 10 & & $768,856,803$ \\
\hline Control 11 & & $875,378,967$ \\
\hline Control 12 & & $667,712,201$ \\
\hline Control 13 & & $842,759,887$ \\
\hline
\end{tabular}

\begin{tabular}{|c|c|c|}
\hline \multicolumn{3}{|c|}{ BE4 edited Progeny } \\
\hline Sample & Gender & Raw Read Counts \\
\hline BE4 1 & & $721,100,551$ \\
\hline BE4 2 & 0 & $792,525,208$ \\
\hline BE4 3 & & $764,834,081$ \\
\hline BE4 4 & & $952,740,731$ \\
\hline BE4 5 & & $743,607,260$ \\
\hline BE4 6 & 1 & $905,084,101$ \\
\hline BE4 7 & $\sigma^{\prime}$ & $798,497,190$ \\
\hline BE4 8 & & $686,497,626$ \\
\hline BE4 9 & & $1,013,557,705$ \\
\hline
\end{tabular}


Supplementary Table 2 SNVs identified in each sample and de novo SNVs of the progeny

\begin{tabular}{|l|c|r|}
\hline \multicolumn{3}{|c|}{ Parents } \\
\hline Sample & Gender & \multicolumn{1}{|c|}{ SNVs } \\
\hline Parent 1 & & 10,542 \\
Parent 2 & & 11,393 \\
Parent 3 & & 11,869 \\
Parent 4 & $\wp$ & 9,91 \\
Parent 5 & + & 10,749 \\
Parent 6 & & 10,149 \\
Parent 7 & & 12,529 \\
Parent 8 & & 11,636 \\
\hline Parent 9 & 0 & 12,42 \\
\hline
\end{tabular}

\begin{tabular}{|c|c|r|r|}
\hline \multicolumn{4}{|c|}{ ABE edited Progeny } \\
\hline Sample & Gender & \multicolumn{1}{|c|}{ SNVs } & de novo SNV ${ }^{1}$ \\
\hline ABE 1 & & 10,997 & 67 \\
ABE 2 & & 10,303 & 100 \\
ABE 3 & + & 9,435 & 73 \\
ABE 4 & & 11,012 & 72 \\
ABE 5 & & 10,984 & 82 \\
\hline ABE 6 & & 12,246 & 138 \\
ABE 7 & & 11,934 & 226 \\
ABE 8 & \multirow{2}{*}{ ABE 9 } & 13,275 & 143 \\
ABE 10 & & 12,593 & 153 \\
ABE 11 & & 12,457 & 128 \\
\hline ABE 12 & \multirow{2}{*}{ N/A } & 12,928 & 155 \\
ABE 13 & 12,344 & 150 \\
\hline \multicolumn{2}{|c}{} \\
\hline
\end{tabular}

\begin{tabular}{|c|c|c|c|}
\hline \multicolumn{4}{|c|}{ Control Progeny } \\
\hline Sample & Gender & SNVs & de novo SNVs ${ }^{1}$ \\
\hline Control 1 & & 11,503 & 95 \\
\hline Control 2 & & 11,139 & 90 \\
\hline Control 3 & + & 10,525 & 93 \\
\hline Control 4 & & 10,309 & 86 \\
\hline Control 5 & & 12,873 & 147 \\
\hline Control 6 & & 12,602 & 164 \\
\hline Control 7 & & 13,467 & 133 \\
\hline Control 8 & & 13,684 & 205 \\
\hline Control 9 & 0 & 13,321 & 105 \\
\hline Control 10 & & 12,669 & 169 \\
\hline Control 11 & & 12,655 & 146 \\
\hline Control 12 & & 13,273 & 111 \\
\hline Control 13 & & 13,314 & 172 \\
\hline
\end{tabular}

\begin{tabular}{|c|c|c|c|}
\hline \multicolumn{4}{|c|}{ BE4 edited Progeny } \\
\hline Sample & Gender & SNVs & de novo SNVs ${ }^{1}$ \\
\hline BE4 1 & & 11,083 & 464 \\
\hline BE4 2 & 9 & 11,038 & 106 \\
\hline BE4 3 & & 10,845 & 174 \\
\hline BE4 4 & & 12,455 & 235 \\
\hline BE4 5 & & 12,85 & 170 \\
\hline BE4 6 & & 12,385 & 164 \\
\hline BE4 7 & 0 & 12,735 & 200 \\
\hline BE4 8 & & 13,056 & 238 \\
\hline BE4 9 & & 12,319 & 241 \\
\hline
\end{tabular}

${ }^{1}$ SNVs identified at the target locus are not included 
bioRxiv preprint doi: https://doi.org/10.1101/731927; this version posted August 12,2019 . The copyright holder for this preprint (which was not certified by peer review) is the author/funder. All rights reserved. No reuse allowed without permission.

Supplementary Table 3 Indels identified in each sample and de novo indels of the progeny

\begin{tabular}{|l|c|r|r|r|}
\hline \multicolumn{5}{|c|}{ Parents } \\
\hline Sample & Gender & Lumpy indels & GATK indels & total indels \\
\hline Parent 1 & & 172 & 7,957 & 8,129 \\
Parent 2 & & 168 & 8,414 & 8,582 \\
Parent 3 & & 151 & 8,188 & 8,339 \\
Parent 4 & 0 & 181 & 8,430 & 8,611 \\
Parent 5 & + & 173 & 8,012 & 8,185 \\
Parent 6 & & 155 & 8,138 & 8,293 \\
Parent 7 & & 148 & 7,936 & 8,084 \\
Parent 8 & & 157 & 8,523 & 8,680 \\
\hline Parent 9 & 0 & 167 & 9,400 & 9,567 \\
\hline
\end{tabular}

\begin{tabular}{|c|c|c|c|c|c|c|c|}
\hline \multicolumn{8}{|c|}{ ABE edited Progeny } \\
\hline Sample & Gender & Lumpy indels & GATK indels & total indels & de novo Lumpy indels ${ }^{1}$ & de novo GATK indels ${ }^{1}$ & total de novo indels ${ }^{1}$ \\
\hline ABE 1 & & 166 & 9,054 & 9,220 & 18 & 162 & 180 \\
\hline ABE 2 & & 171 & 9,447 & 9,618 & 24 & 185 & 209 \\
\hline ABE 3 & 9 & 196 & 9,294 & 9,490 & 41 & 196 & 237 \\
\hline ABE 4 & & 179 & 8,603 & 8,782 & 27 & 119 & 146 \\
\hline ABE 5 & & 163 & 8,435 & 8,598 & 24 & 155 & 179 \\
\hline ABE 6 & & 179 & 9,040 & 9,219 & 28 & 189 & 217 \\
\hline ABE 7 & & 206 & 9,157 & 9,363 & 70 & 249 & 319 \\
\hline ABE 8 & $\lambda$ & 171 & 9,128 & 9,299 & 33 & 174 & 207 \\
\hline ABE 9 & $\delta$ & 153 & 9,131 & 9,284 & 19 & 195 & 214 \\
\hline ABE 10 & & 164 & 9,180 & 9,344 & 24 & 171 & 195 \\
\hline ABE 11 & & 149 & 8,590 & 8,739 & 18 & 165 & 183 \\
\hline ABE 12 & $N / A$ & 155 & 8,112 & 8,267 & 28 & 175 & 203 \\
\hline ABE 13 & $\mathrm{~N} / \mathrm{A}$ & 169 & 8,671 & 8,840 & 17 & 121 & 138 \\
\hline
\end{tabular}

\begin{tabular}{|c|c|c|c|c|c|c|c|}
\hline \multicolumn{8}{|c|}{ BE4 edited Progeny } \\
\hline Sample & Gender & Lumpy indels & GATK indels & total indels & de novo Lumpy indels ${ }^{1}$ & de novo GATK indels ${ }^{1}$ & total de novo indels ${ }^{1}$ \\
\hline BE4 1 & & 162 & 8,869 & 9,031 & 13 & 141 & 154 \\
\hline BE4 2 & 운 & 164 & 9,093 & 9,257 & 25 & 160 & 185 \\
\hline BE4 3 & & 165 & 9,048 & 9,213 & 10 & 119 & 129 \\
\hline BE4 4 & & 171 & 9,017 & 9,188 & 25 & 206 & 231 \\
\hline BE4 5 & & 159 & 9,241 & 9,400 & 16 & 173 & 189 \\
\hline BE4 6 & & 142 & 8,712 & 8,854 & 20 & 188 & 208 \\
\hline BE4 7 & 0 & 159 & 9,225 & 9,384 & 21 & 216 & 237 \\
\hline BE4 8 & & 158 & 8,565 & 8,723 & 26 & 189 & 215 \\
\hline BE4 9 & & 181 & 9,389 & 9,570 & 40 & 209 & 249 \\
\hline
\end{tabular}

\begin{tabular}{|c|c|c|c|c|c|c|c|}
\hline \multicolumn{8}{|c|}{ Control Progeny } \\
\hline Sample & Gender & Lumpy indels & GATK indels & total indels & de novo Lumpy indels & de novo GATK indels & total de novo indels \\
\hline Control 1 & & 175 & 8,648 & 8,823 & 35 & 115 & 150 \\
\hline Control 2 & 0 & 167 & 8,423 & 8,590 & 19 & 109 & 128 \\
\hline Control 3 & + & 164 & 8,691 & 8,855 & 27 & 139 & 166 \\
\hline Control 4 & & 208 & 8,776 & 8,984 & 67 & 154 & 221 \\
\hline Control 5 & & 149 & 9,256 & 9,405 & 17 & 200 & 217 \\
\hline Control 6 & & 206 & 8,885 & 9,091 & 63 & 204 & 267 \\
\hline Control 7 & & 203 & 9,000 & 9,203 & 59 & 166 & 225 \\
\hline Control 8 & & 144 & 8,800 & 8,944 & 18 & 173 & 191 \\
\hline Control 9 & $0^{1}$ & 157 & 8,754 & 8,911 & 33 & 150 & 183 \\
\hline Control 10 & & 156 & 9,165 & 9,321 & 20 & 166 & 186 \\
\hline Control 11 & & 153 & 9,232 & 9,385 & 22 & 193 & 215 \\
\hline Control 12 & & 187 & 8,944 & 9,131 & 38 & 164 & 202 \\
\hline Control 13 & & 144 & 9,418 & 9,562 & 12 & 165 & 177 \\
\hline
\end{tabular}

\footnotetext{
${ }^{1}$ indels identified at the target locus are not included
} 
Supplementary Table 4 Location of the four predicted off-target sites overlapping with de novo SNVs. The samples harboring the same SNV are grouped into overlapping groups.

\begin{tabular}{|ccc|c|c|c|c|}
\hline \multicolumn{3}{|c|}{ off-target coordinate } & \multicolumn{3}{c|}{ SNVs groups overlapping with the off-target site } \\
\hline chr & start & stop & Overlap 1 & Overlap 2 & Overlap 3 & Overlap 4 \\
\hline chr11 & 60377453 & 60377475 & ABE 5 & BE4 7 & BE4 1 & BE4 8 \\
& & & & BE4 8 & BE4 3 & \\
& & & & BE4 7 & \\
\hline
\end{tabular}

\begin{tabular}{|lll|l|l|l|l|}
\hline chr13 & 114169949 & 114169971 & ABE 1 & & & \\
\hline \multicolumn{7}{|c|}{} \\
\hline chr9 & 40294521 & 40294543 & ABE 2 & ABE 3 & & \\
& & ABE 4 & ABE 4 & & \\
& & & ABE 6 & & \\
& & & ABE 7 & & \\
\hline
\end{tabular}


Supplementary Table 5: Characterization of de novo indels.

\begin{tabular}{|l|r|r|r|}
\cline { 2 - 4 } \multicolumn{1}{c|}{} & \multicolumn{1}{c|}{ GATK indels } \\
\cline { 2 - 4 } \multicolumn{1}{c|}{} & $\begin{array}{c}\text { ABE edited } \\
\text { mean (+/- s.d.) }\end{array}$ & $\begin{array}{c}\text { BE4 edited } \\
\text { mean (+/-s.d.) }\end{array}$ & \multicolumn{1}{c|}{$\begin{array}{c}\text { Control } \\
\text { mean (+/-s.d.) }\end{array}$} \\
\hline deletions & $24 \%(+/-6.1)$ & $27 \%(+/-6.5)$ & $29 \%(+/-4.1)$ \\
insertions & $76 \%(+/-6.1)$ & $73 \%(+/-6.5)$ & $71 \%(+/-4.1)$ \\
\hline avg. deletion size (bp) & $2.7(+/-0.8)$ & $2.9(+/-0.7)$ & $2.9(+/-0.5)$ \\
\hline avg. insertion size (bp) & $15.2(+/-3.4)$ & $13.3(+/-2.5)$ & $12(+/-3.1)$ \\
\hline
\end{tabular}

\begin{tabular}{|l|r|r|r|}
\cline { 2 - 4 } \multicolumn{1}{c|}{} & \multicolumn{1}{c|}{ LUMPY indels } \\
\cline { 2 - 4 } \multicolumn{1}{c|}{} & \multicolumn{1}{c|}{$\begin{array}{c}\text { ABE edited } \\
\text { mean (+/-s.d.) }\end{array}$} & $\begin{array}{c}\text { BE4 edited } \\
\text { mean (+/-s.d.) }\end{array}$ & \multicolumn{1}{c|}{$\begin{array}{c}\text { Control } \\
\text { mean (+/-s.d.) }\end{array}$} \\
\hline deletions & $64.1 \%(+/-13.2)$ & $57.6 \%(+/-10.1)$ & $64.4 \%(+/-18.6)$ \\
duplications & $0.7 \%(+/-1.7)$ & $0 \%(+/-0)$ & $0.6 \%(+/-1.5)$ \\
invertion & $0.6 \%(+/-2.3)$ & $0 \%(+/-0)$ & $0.8 \%(+/-1.7)$ \\
complex & $34.6 \%(+/-11.5)$ & $42.4 \%(+/-10.1)$ & $34.1 \%(+/-18.4)$ \\
\hline avg. deletion size (bp) & $672(+/-332)$ & $1,001(+/-843)$ & $2,218(+/-4,343)$ \\
\hline avg. duplication size (bp) & $123,900(+/-27,788)$ & $\mathrm{N} / \mathrm{A}$ & $52,376(+/-73,358)$ \\
\hline avg. invertion size (bp) & $115(+/-0)$ & $\mathrm{N} / \mathrm{A}$ & $12,516(+/-17,452)$ \\
\hline
\end{tabular}

